\title{
ON PERIODIC MAPS WHICH RESPECT A SYMPLECTIC STRUCTURE
}

\author{
C. B. THOMAS
}

The lacunae in our knowledge of the coefficients have made direct computations in the homology theory $\Omega_{*}^{\mathrm{Sp}}(X, A)$ almost impossible. This note sidesteps the difficulty by exploiting the relationship between the theories $\mathrm{KO}^{*}$ and $\Omega_{\mathrm{Sp}}^{*}$, which corresponds to that between $\mathrm{KU}^{*}$ and $\Omega_{U}^{*}$, and which is fully discussed in Chapter II of [1]. In this way we are able to calculate the additive orders of some important elements in $\Omega_{*}^{\mathrm{Sp}}\left(\boldsymbol{Z}_{p}\right)$ ( $p$ prime), the module of principal $\boldsymbol{Z}_{p}$-manifolds with compatible weak symplectic structure. Perhaps the most interesting aspect of the computation is the difference between the cases $p$ odd and $p$ equals 2 , reflecting the presence of 2 -torsion in $\Omega_{*}^{\mathrm{Sp}}$.

I am grateful to Elmer Rees for discussing this problem with me and for showing me his version of the computations in [2] for real projective space.

Notation. Let $\mathrm{KU}(), \mathrm{KO}(\mathrm{)}$ ) and $\mathrm{KSp}($ ) be the cohomology functors defined by unitary, orthogonal and symplectic bundles respectively. There are natural operators

$$
\mathrm{KO}(X) \underset{p}{\stackrel{i}{\rightleftharpoons}} \mathrm{KU}(X) \underset{q}{\stackrel{j}{\rightleftharpoons}} \mathrm{KSp}(X)
$$

and complex conjugation $c: \mathrm{KU}(X) \rightarrow \mathrm{KU}(X)$. These satisfy the relations

$$
\begin{array}{lll}
p i=2 & q j=1+c & p c=p \\
i p=1+c & j q=2 & j c=j .
\end{array}
$$

This notation will be used below without further comment.

The relation between K-theory and bordism. As in the SO- and $\mathrm{U}$-bordism theories there is a natural isomorphism between $\Omega_{n}^{\mathrm{Sp}}\left(\boldsymbol{Z}_{p}\right)$ and $\Omega_{n}^{\mathrm{Sp}}\left(K\left(\boldsymbol{Z}_{p}, 1\right)\right)$, defined by classifying a principal $\boldsymbol{Z}_{p}$-manifold as a principal $\boldsymbol{Z}_{p}$-bundle. Let $T$ be the weakly symplectic $\boldsymbol{Z}_{p}$-action which defines the lens space

$$
\begin{gathered}
L^{4 n-1}(p ; 1, \cdots, 1)=L_{p}^{4 n-1} . \\
2 n \text { times }
\end{gathered}
$$

Received by the editors October 21, 1968. 
When $p$ equals $2, T$ reduces to the antipodal map $A$. Under the isomorphism above $\left[T, S^{4 n-1}\right]$ corresponds to the inclusion of $L_{p}^{4 n-1}$ in the infinite join, which is the standard $K\left(Z_{p}, 1\right)$. In [1] Conner and Floyd prove that the first cobordism Pontrjagin class $p_{1}: \tilde{K} \mathrm{Sp}(X)$ $\rightarrow \Omega_{\mathrm{Sp}}^{4}(X)$ is a monomorphism. If $h_{H}$ is the canonical symplectic line bundle over $H P(n)$, let $\nu \in \tilde{\mathrm{K}} \mathrm{Sp}(H P(n))$ denote the class of $\left(h_{H}-1\right)$. The image of $\nu$ under $p_{1}$ is the cobordism class of the inclusion $u: H P(n) \hookrightarrow H P(\infty)$. Let $\pi: L_{p}^{4 n+3} \rightarrow H P(n)$ be the projection of the natural fibration, and consider the commutative diagram:

$$
\begin{aligned}
& \underset{\uparrow \pi^{!}}{\tilde{\mathrm{K}} \operatorname{Sp}\left(L_{p}^{4 n+3}\right)} \underset{p_{1}}{\underset{\mathrm{Sp}}{\longrightarrow}} \underset{\Omega_{\mathrm{Sp}}^{4}\left(L_{p}^{4 n+3}\right)}{\stackrel{\sim}{\vec{D}}} \Omega_{4 n-1}^{\mathrm{Sp}}\left(L_{p}^{4 n+3}\right) \\
& \tilde{\mathrm{K}} \mathrm{Sp}(H P(n)) \underset{p_{1}}{\longrightarrow} \Omega_{\mathrm{Sp}}^{4}(H P(n)) \text {. }
\end{aligned}
$$

The isomorphism $D$ on the right is Poincare duality.

$$
p_{1} \pi ! \nu=\left\{u \pi: L_{p}^{4 n+3} \rightarrow H P(\infty)\right\} .
$$

Regard $H P(\infty)$ as a submanifold of codimension 4 in itself. Duality, which is a variant of the Thom representation theorem for bordism, then implies that the final image of $\pi^{\prime} \nu$ is the bordism class $\left\{L_{p}^{4 n-1} \hookrightarrow L_{p}^{4 n+3}\right\}$. Finally an application of the cellular approximation theorem shows that the map

$$
V_{*}: \Omega_{4 n-1}^{\mathrm{Sp}}\left(L_{p}^{4 n+3}\right) \rightarrow \Omega_{4 n-1}^{\mathrm{Sp}}\left(Z_{p}\right)
$$

is also a monomorphism. This proves

LEMMA. The order of $\left[T, S^{4 n-1}\right]$ in $\Omega_{4 n-1}^{\mathrm{Sp}}\left(\boldsymbol{Z}_{p}\right)$ ( $p$ any prime) is equal to the order of $\pi^{!} \nu$ in $\tilde{\mathrm{K}} \mathrm{Sp}\left(L_{p}^{4 n+3}\right)$.

Orders of the bordism classes.

Proposition 1. For $p$ odd $\left[T, S^{4 n-1}\right]$ has additive order $p^{k+1}$ in $\Omega_{4 n-1}^{\mathrm{Sp}}\left(\boldsymbol{Z}_{p}\right)$, where $k(p-1) \leqq 2 n+1<(k+1)(p-1)$.

Proof. Let $\pi_{0}: L_{p}^{4 n+3} \rightarrow \boldsymbol{C} P(2 n+1)$ be the natural fibration, and consider the composition

$$
\frac{L_{p}^{4 n+3} \underset{S^{1}}{\stackrel{\pi_{0}}{\longrightarrow} C P(2 n+1) \underset{S^{2}}{\rightarrow} H P(n) .}}{\pi}
$$

If $h_{C}$ is the canonical line bundle over $C P(2 n+1)$, denote the class 
of $\left(h_{C}-1\right)$ in $\tilde{\mathrm{K}} \mathrm{U}(\boldsymbol{C} P(2 n+1))$ by $\mu$, and the pullback of $\mu$ along $\pi_{0}$ by $\sigma$. Since the operator $j$ commutes with continuous maps, and the pullback of $\nu$ is $j \mu$,

$$
\pi^{\prime} \nu=j \pi_{0}^{\mathfrak{1}} \mu=j \sigma
$$

The same argument as that used by $\mathrm{T}$. Kambe in [3], only with $\tilde{\mathrm{K}} \mathrm{Sp}$ replacing $\tilde{\mathrm{K}}$, shows that $\tilde{\mathrm{K}} \mathrm{Sp}\left(L_{p}^{4 n+3}\right)$ consists of $p$-torsion. (The top dimensional cell cannot introduce a factor $Z_{2}$, since $4 n+3$ $\not \equiv 5 \bmod 8$.) Hence for the pair of operators $(j, q)$, where $j q$ is multiplication by $2, q$ is a monomorphism and $j$ an epimorphism. Composing in the reverse order, $q j(\sigma)=(1+c) \sigma$. From the properties of the complex line bundle over $\boldsymbol{C} P(2 n+1)$, and the naturality of $q$ with respect to $\pi_{0}^{!}$, it follows that

$$
q(j \sigma)=\sigma^{2}-\sigma^{3}+\sigma^{4}-\cdots
$$

T. Kambe (op. cit.) has computed the group $\tilde{\mathrm{K}} \mathrm{U}\left(L_{\mathrm{p}}^{4 n+3}\right)$, and it follows from his calculations that $q(j \sigma)$, and hence $j \sigma$, have order $p^{k+1}$, where $k(p-1) \leqq 2 n+1<(k+1)(p-1)$. Proposition 1 now follows from the Lemma.

The situation when $p$ equals 2 is more complicated, since in the presence of 2-torsion it is not clear that $j$ is an epimorphism. Also in this special case we replace the symbol $T$ by $A$.

Proposition 2.(i) $\left[A, S^{8 n+3}\right]$ has order $2^{4 n+3}$ in $\Omega_{8 n+3}^{\mathrm{Sp}}\left(Z_{2}\right)$, and (ii) $\left[A, S^{8 n+7}\right]$ has order $2^{4 n+4}$ in $\Omega_{8 n+7}^{\mathrm{Sp}}\left(Z_{2}\right)$.

Proof. The projection $\pi$ reduces to the fibration of projective spaces $R P(4 n+3) \rightarrow H P(n)$ and $h_{H}$ pulls back to the canonical line bundle $h_{R}$ over $R P(4 n+3)$ regarded as a symplectic bundle. Denote the class of $\left(h_{R}-1\right)$ in $\tilde{\mathrm{KO}}(R P(4 n+3))$ by $\lambda$, when $\pi^{\prime} \nu=j i(\lambda)$. It is not obvious that $j i(\lambda)$ is always a generator of $\tilde{\mathrm{KSp}}(R P(4 n+3))$, but from Bott periodicity and the fact that $i \lambda$ generates $\tilde{\mathrm{K}} U(R P(4 n+3))$, it follows that it is enough to look at the cokernel of the forgetful map $p$. Consider the long exact sequence of groups associated with the pair $(\mathrm{U}, \mathrm{O})$ having quotient homotopically equivalent to $\Omega \mathrm{O}$.

$$
\begin{aligned}
\cdots & \rightarrow \tilde{\mathrm{K}} O^{3} X \stackrel{\delta}{\rightarrow} \tilde{\mathrm{K}} \mathrm{O}^{2} X \rightarrow \tilde{\mathrm{K}} \mathrm{U}^{4} X \stackrel{p}{\rightarrow} \tilde{\mathrm{K}} O^{4} X \\
& \rightarrow \tilde{\mathrm{K}} \mathrm{O}^{3} X \stackrel{\delta}{\rightarrow} \tilde{\mathrm{K}} \mathrm{U}^{5} X \rightarrow \cdots
\end{aligned}
$$

From the computations of M. Fujii we have the particular cases: 


$$
\begin{array}{ll}
X=R P(8 n+3), & \rightarrow \boldsymbol{Z} \rightarrow \boldsymbol{Z}_{2}+\boldsymbol{Z}_{2} \rightarrow \boldsymbol{Z}_{2^{4 n+1}} \rightarrow \boldsymbol{Z}_{2^{4 n}} \stackrel{0}{\rightarrow} \boldsymbol{Z} \rightarrow \boldsymbol{Z} \rightarrow \\
X=R P(8 n+7), & \rightarrow \boldsymbol{Z} \rightarrow 0 \rightarrow \boldsymbol{Z}_{2^{4 n+3}} \stackrel{\sim}{\longrightarrow} \boldsymbol{Z}_{2^{4 n+3}} \stackrel{0}{\rightarrow} \boldsymbol{Z} \rightarrow \boldsymbol{Z} \rightarrow .
\end{array}
$$

In both cases the map $p$ is an epimorphism. Since we are only interested in the magnitudes of the various groups, we can use periodicity to replace the sequence $\left({ }^{*}\right)$ with that associated with the pair $(\mathrm{U}, \mathrm{Sp})$, provided we shift the dimensions by 4 . It follows that $j$ is also an epimorphism, and that $j i(\lambda)$ generates the cyclic group $\tilde{\mathrm{K}} \mathrm{Sp}(R P(4 n+3))$. Proposition 2 now follows from the Lemma.

Additional remarks. At least when $p$ is odd, it is possible to show that the submodule of $\tilde{\Omega}_{*}^{\mathrm{Sp}}\left(\boldsymbol{Z}_{p}\right)$ generated by $\left\{\left[T, S^{4 n-1}\right], n \geqq 1\right\}$ is proper. The spectral sequence for $\tilde{\Omega}_{*}^{S p}\left(\boldsymbol{Z}_{p}\right)$ has nonzero terms at the $E_{r, s}^{2}$ level only when $s \equiv 0 \bmod 4, r \equiv 1 \bmod 2$, and hence collapses. This is a consequence of the isomorphism of $\tilde{\Omega}_{*}^{\mathrm{Sp}} \otimes Z[1 / 2]$ with $\Omega_{*}^{\text {SO }} \otimes Z[1 / 2]$ as $Z[1 / 2]$ polynomial algebras, [4]. It follows that the edge homormorphism from $\Omega_{*}^{\mathrm{Sp}}\left(\boldsymbol{Z}_{p}\right)$ to $H_{*}\left(\boldsymbol{Z}_{p}, \boldsymbol{Z}\right)$ is an epimorphism, and that our construction has failed to pick up a complete set of module generators.

As in the oriented, and in contrast to the unitary theory, the structure of $\Omega_{*}^{\mathrm{Sp}}\left(\boldsymbol{Z}_{2}\right)$ does not follow as a special case from that of $\widetilde{\Omega}_{*}^{\mathrm{Sp}}\left(\boldsymbol{Z}_{p}\right)$. The general pattern of Proposition 1 would suggest that $\left[A, S^{8 n+3}\right]$ and $\left[A, S^{8 n+7}\right]$ have orders $2^{4 n+3}$ and $2^{4 n+5}$ respectively. In the second case the predicted order differs by a factor of 2 from the actual order; no doubt this is a consequence of the 2-torsion structure of $\Omega_{*}^{\mathrm{Sp}}$ itself.

\section{REFERENCES}

1. P. E. Conner and E. E. Floyd, The relation of cobordism to K-theories, SpringerVerlag, New York, 1966.

2. M. Fujii, KO-groups of projective spaces, Osaka J. Math. 4 (1967), 141-149.

3. T. Kambe, The structure of K-rings of the lens space and their applications, J. Math. Soc. Japan 18 (1966), 135-146.

4. S. P. Novikov, Homotopy properties of Thom complexes, Mat. Sb. (N.S.) 57 (92) (1962), 407-442.

UNIVERSITY OF HULL 